\title{
THE BLACK SILICON METHOD II: THE EFFECT OF MASK MATERIAL AND LOADING ON THE REACTIVE ION ETCHING OF DEEP SUICON TRENCHES
}

\author{
Henri Jansen, Meint de Boer, Johannes Burger, Rob Legtenberg, and Miko Elwenspoek \\ MESA Research Institute, University of Twente, P.O. Box 217, 7500 AE Enschede, The Netherlands
}

Phone: X-31-53-894373; Secr: X-31-53-892751; Fax: X31-53-309547

Very deep trenches in Si with smooth controllable profiles are etched using a fluorine-based Reactive Ion Etcher (RIE). The effect of various mask materials and loading on the profile is examined using the Black Silicon Method. It is found that most metal layers have an almost infinite selectivity. When the aspect ratio of the trenches is beyond five, RIE lag is found to be an important effect. Evidence is found that this effect is caused by the bowing of incoming ions by the electrical field.

\section{INTRODUCTION}

We have recently demonstrated that trenches (up to 200 $\mu \mathrm{m}$ ) with high aspect ratios (up to 10 ) in $\mathrm{Si}$ are etched at high etch rates (up to $5 \mu \mathrm{m} / \mathrm{min}$ ) and low ion energies (15-90eV) using a fluorine-based RIE plasma (SF6/$\mathrm{O} 2 / \mathrm{CHF} 3$ ) and the Black Silicon Method (BSM) [1,2].

The low ion energy prevents substrate damage (electronics), mask erosion (the selectivity to metal masks is practically infinite), and makes it easy to change the profile of the trench.

In the BSM the forming of "grass" or black Si is used to find the vertical wall regime and after this the profile can be altered by changing the plasma chemistry (power, pressure, or flows) with the help of diagrams.

Etching rates and profiles have been observed to depend on feature size (i.e. aspect ratio dependent etching: ARDE) and pattern density (i.e. microloading) for $\mathrm{Si}, \mathrm{SiO}$, polymers, metals, and III-V's, also referred to as microscopic nonuniformity [3].

In this article a comprehensive overview of the BSM will be given [1]. The effect of selectivity, loading, and mask materials on deep $\mathrm{Si}$ etching, together with some important aspects (ion bowing, RIE lag, etch rate, and uniformity) will be discussed by using the BSM.

\section{THE BLACK SILICON METHOD}

In an $\mathrm{SF} 6 / \mathrm{O} 2 / \mathrm{CHF} 3$ plasma, each gas has a known specific function and influence, so the etched profile is easily controlled just by changing the flow rate of one of these gases [1,2]. In such a plasma SF6 produces the $\mathrm{F}^{*}$ radicals for the chemical etching of the Si forming the volatile $\mathrm{SiF} 4, \mathrm{O} 2$ creates the $\mathrm{O}^{*}$ radicals to passivate the $\mathrm{Si}$ surface with $\mathrm{SiOxFy}$, and $\mathrm{CHF}^{3}$ (or $\mathrm{SF6}$ ) is the source of $\mathrm{CFx}+$ (or $\mathrm{SFx}+$ ) ions, responsible for the removal of the SiOxFy layer at the bottom of the etching trenches forming the volatile COxFy (or SOxFy

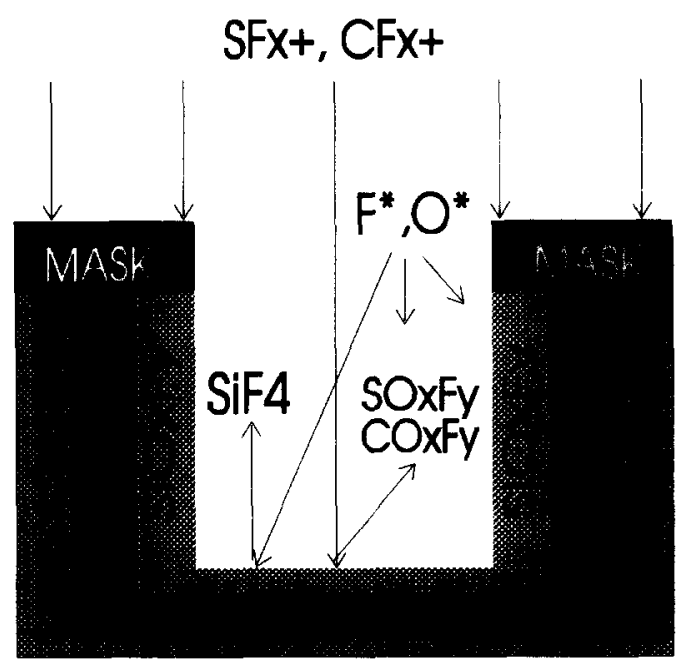

Diagram 1: The SF6/O2/CHF3 chemistry.

(diagram 1).

Of course, it is possible to continue this paper by giving the recipe for the RIE of silicon giving a vertical wall profile and show the influence of the various parameters on this profile such as power, pressure, and gas flow. However, this information can't be used in other laboratories, especially when they have a different reactor. For this reason a stronger tool is used to find the vertical profile regime. This method uses the fact that the silicon is turned black when the vertical wall recipe is found. From now on, this method will be called the "Black Silicon Method" (BSM). Another method using response surface methodology (RSM) has previously been described by the authors [2]; it includes information about the equipment and the actual recipe. Before the BSM is formulated, the reason for this effect will be explained and a way to get rid of this blackening will be described. 


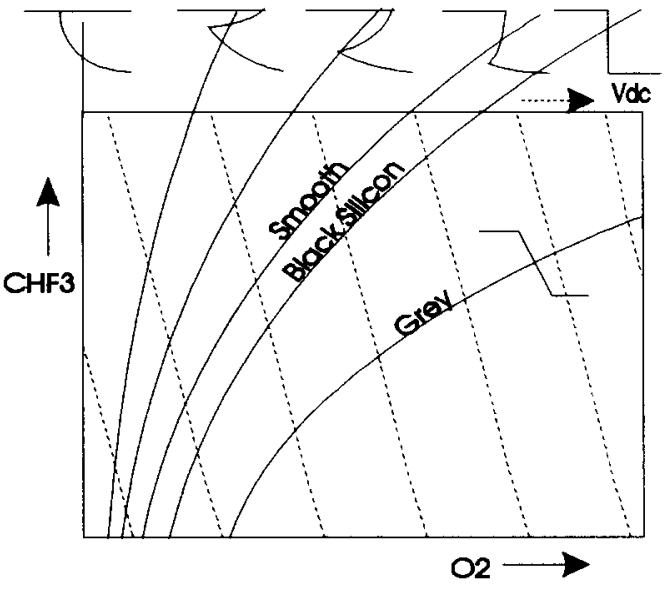

Diagram 2a: The influence of the flow on the profile.

\section{The origin of black silicon:}

As stated in the above there is a constant competition between the fluorine radicals that etch and the oxygen radicals that passivate the silicon. At a certain oxygen content there is such a balance between the etching and the passivation that a nearly vertical wall results. At the same moment native oxide, dust, et cetera will act as micro masks and, because of the directional etching, spikes will appear. These spikes consist of a silicon body with a thin passivating siliconoxyfluoride skin. They will become higher in time and, depending on the etch rate, they will exceed the wavelength of incoming light after some time. This light will be "catched" in the areas between the spikes and can't leave the silicon surface anymore. So, all the light is collected by the etching surface and it is turned into black. The origin of micro masks is caused by native oxide, dust, and so on which is already on the wafer before etching. But, it is also formed during the etching because siliconoxide particles coming from the plasma are adsorbing at the silicon surface or because of the oxidation of the silicon surface together with the angle dependent ion etching of this oxide layer. Another source of particles during etching which will act as micro masks is the resputtering of mask material due to imparting ions.

Preventing black silicon: It is possible to forbid spikes from forming by constantly underetching the micro masks isotropically or etching the features with a slightly negative undercut. The isotropic solution makes only sense when it is used as a post etch, because otherwise the feature density is limited. On the other hand, the negative underetching is an excellent way to control the smoothness of the substrate surface barely limiting the feature size density. In this study the

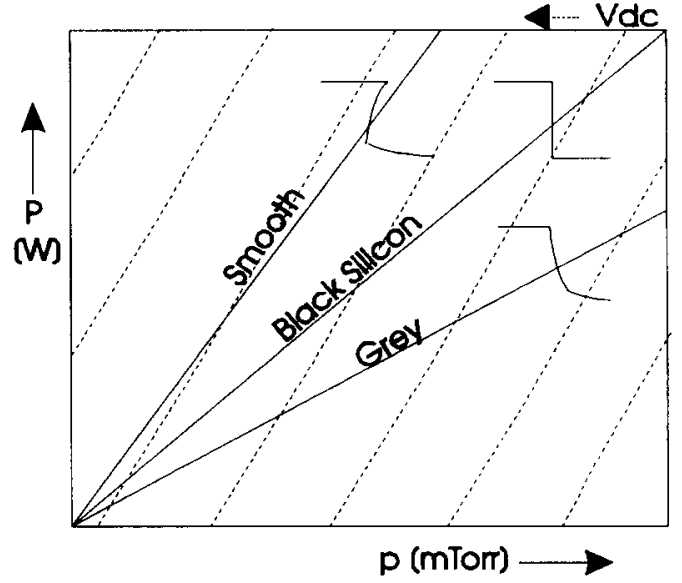

Diagram 2b: The influence of the power and pressure on the profile.

addition of CHF3 to an SF6/O2 plasma is described and its ability to prevent grass.

The Black Silicon Method: In this section, an easy way to find the vertical wall regime is described with the help of the information already given. A more or less general tool is reached in which the recipe for any RIE system can be found just by fulfilling the sequence written down below. As can be concluded from point 3 of this sequence purely vertical walls can be achieved for any pressure, power, O2-, CHF3-, or SF6-flow. This is an important conclusion because now we are able to create any d.c. self-bias we want without changing the profile. For instance, it is possible to develop very low bias voltages $(<20 \mathrm{eV})$ at the higher pressures giving very high mask selectivity, maintaining the profile. In such cases the etched silicon bottom and the sidewalls are nearly perfect. It is also observed in diagram $2 a$ that a vertical wall profile is found for zero CHF3 flow. This means that the passivation with siliconoxyfluorides at the sidewalls is more likely than the passivation with a fluorocarbon layer, although it is still possible that at different pressure, loading, et cetera, the fluorocarbon layer is more pronounced.. Also, the observation that increasing the oxygen flow gives rise to a more positively tapered profile is a strong indication that siliconoxyfluoride is the sidewall passivator. Auger analysis showed that indeed the sidewalls are covered with silicon oxide; there is no carbonic species found [2].

The Black Silicon Method is tested for three different RIE systems. Most experiments are performed with a plan parallel plate reactor "plasmafab 340" from the STS company [2] and a second plan parallel plate single wafer reactor "plasmatherm 500" showed identical results. A third system, the hexode "AME- 
$8100^{\prime \prime}$ from Applied Materials, is used for the batch fabrication of silicon wafers and is also able to achieve vertical profiles. However, the etch rates are approximately one order in magnitude lower than for the single wafer etchers and for this reason less powerful.

Although the Black Silicon Method was originally developed for silicon trench etching, it is found that this method works for siliconoxynitride and polymer trench etching as well. Although the appearance of a polymer surface after anisotropic etching is not black but rather diffuse the mechanism is the same. For this reason a more general name for this method is chosen; the Black Substrate Method.

\section{The formulation of the Black Silicon Method}

1. Place a piece of silicon in the reactor and adjust the preferred power and pressure for an $\mathrm{SF} 6 / \mathrm{O} 2$ plasma. Etch ca. 1 micron of silicon, open the process chamber, and look if the silicon is black. If not, do the same again but increase the oxygen flow. Proceed with this sequence until the wafer is black. Increasing the oxygen too much, still will give rise to black-, or better grey-, silicon since there exists a positively tapered profile without any underetching (diagram 2a).

2. After the black silicon regime is found add some $\mathrm{CHF} 3$ to the mixture and increase this flow until the wafer is clean again. Too much CHF3 will make the profiles isotropic (and smooth) because the CFx species are scavenging the oxygen radicals which are needed for the blocking layer.

3. Now a wafer with the mask pattern of interest is inserted in the reactor and the etched profile is checked. If necessary, add some extra silicon in the reactor chamber until the exposed silicon area is the same as in step 1 and 2. Increasing the SF6 content will create a more isotropic profile. Adding more oxygen will make the profile positively tapered and extra CHF 3 will make it more negatively tapered (diagram 2a). Adding at the same time $\mathrm{O} 2$ and CHF3 with the correct balance will create very smooth and nearly vertical walls (fig.1, small trenches). Increasing the pressure or decreasing the power will make the profile more positively tapered. In diagram $2 \mathrm{a} / \mathrm{b}$ the influence of the $\mathrm{O} 2 / \mathrm{CHF} 3$ flow and the pressure/power on the profile is given. Increasing at the same time the $\mathrm{O} 2$ - and $\mathrm{CHF}$ 3-flow or power, increasing the $\mathrm{O} 2$ flow while decreasing the pressure, decreasing the pressure and power or CHF3 flow and decreasing the CHF3 flow while increasing the power, will hardly change the profile. However, such a change will increase the d.c. self-bias and a higher d.c. self bias will give the off-normal ions enough energy to etch the sidewalls, thus changing the profile a little.

\section{ION BOWING}

Aspect ratio dependent etching (ARDE) is a collective noun for sidewall bowing, feature size dependency of profiles, and RIE lag. They are well known phenomena observed while etching trenches into a conducting substrate and seem to be strongly correlated by the effect of ion bowing. Ion bowing is caused by the diffraction of ions while entering a trench or by the negative potential of trench walls with respect to the plasma glow resulting in a deflection of these ions to the walls $[4,5]$. These ions are the main etching specimen of the passivating SiOxFy layer, and are controlling the etched profile by their direction. In this section it is made plausible that the physical ion depletion and bowing and not the chemical depletion or other mechanisms are responsible for the phenomena observed [3].

In fig. 1 a typical example of sidewall bowing is observed. In the wider trench, ions are deflected to the walls during their trajectory resulting in a parabolic curvature of the etched wall (i.e. negatively tapered).

The feature size dependency of profiles is also found in fig. 1, where the wider trench is more negatively tapered than the smaller trenches. Ions which enter a small cavity will be less attracted by its nearest wall because its opposite wall is closer than in the case of a wide opening. This wall is neutralising the closest wall and therefore the smaller opening is more pos. tapered.

RIE lag is associated to the effect that smaller trenches are etched slower (pos. lag) or faster (neg. lag) than wider trenches (fig.1). Positive lag may be explained in considering the amount of ions which exist during their trajectory in the trench. During this travel the outer ions will be collected by the negative (conducting) walls, while etching it, and this process will go on until

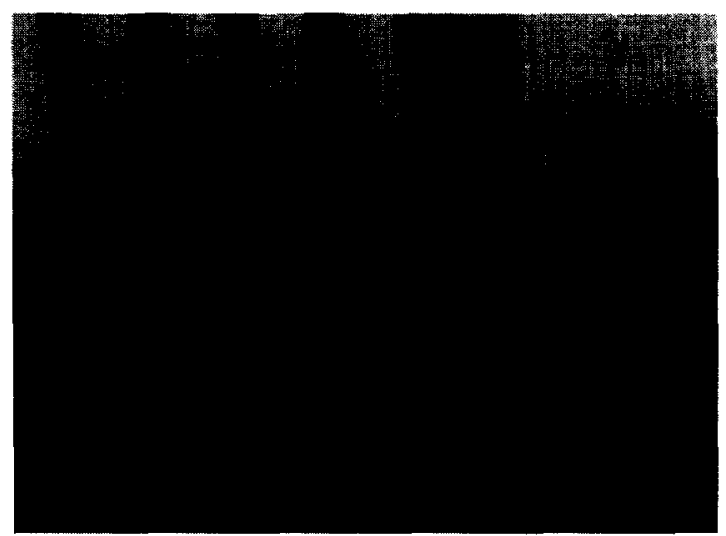

Fig. 1: The influence of ion bowing on the profile. RIE setting: power $=75 \mathrm{~W}$, pressure $=75 \mathrm{mT}$ Trr, $\mathrm{SF} 6 / \mathrm{O} 2 / \mathrm{CHF} 3=28 / 10 / 5 \mathrm{sccm}$. 


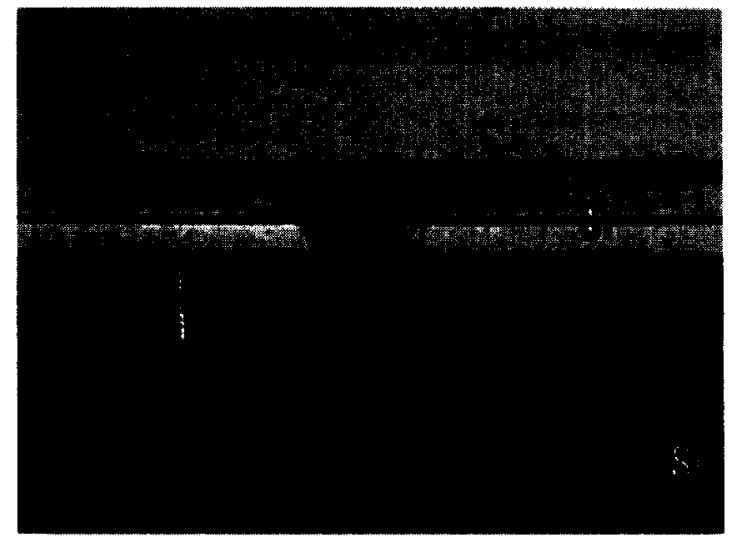

Fig.2: Preventing RIE lag with the help of the BSM. RIE setting: power $=75 \mathrm{~W}$, pressure $=100 \mathrm{mTorr}, \mathrm{SF} 6 / \mathrm{O} 2 / \mathrm{CHF} 3=28 / 15 / 21 \mathrm{sccm}$.

all the ions are captured by the walls. So, at last there will be no ions left to etch the bottom and etching stops in this direction. It is obvious that for the small trenches this "ion depletion" is reached sooner than for the widest because the flux/wallarea ratio is smaller after a certain etch depth (fig. 1). In fact, it can be shown easily that only the relative trench dimensions and not the absolute trench dimensions are controlling the etchdepth and -rate. For a certain plasma setting we observed that the etch rate is decreasing measurable (>10\%) when the depth of the trench is twice the width and is almost zero when the aspect ratio is 10 or more.

The neg. lag seems to be possible when the trenches can collect ions from their non etching (isolating) surrounding [6] or when the etch rate is decreasing because of depletion of etching radicals in the trench. It is also observed in ion inhibitor processes [3], such as the BSM chemistry, but we were not able to create it.

Ion bowing should not change the profile of insulating trenches because this effect would soon be outruled by the positive charging of the walls. Therefore we investigated the etching of polymers and $\mathrm{SiOxNy}$ layers. During etching these layers sidewall bowing and RIE lag were barely observed indicating that the conducting wall can explain the phenomena observed [7]. However, in literature RIE lag is without doubt observed for isolating substrate materials [3]. The reason for this discrepancy is not clear yet.

The effect of RIE lag can be controlled with the help of the Black Silicon Method. In fig. 2 the lag has been reduced by making the $\mathrm{Si}$ trench wall more insulating like, thus increasing the passivator in the plasma. To ensure a certain profile, the ion flux has to be increased at the same time conform the BSM.

\section{MASK MATERIALS}

While etching $\mathrm{Si}$ it is important that the mask material has no or at least a known influence on the plasma chemistry and the etch result. The profile is changing with the etchability, conductivity, and temperature of the mask. The influence of catalytic reactions of the mask material [8] are not observed in our study. The mask should be deposited without complicating the technology (incl. compatibility). In this section a broad spectrum of masks is tested for their influence on the pattern transfer, selectivity, and erosion characteristics with the help of the Black Silicon Method.

Etchability: In general, an etching mask will influence the Si trench profile because the mask is retarding when its profile is not fully vertical. Almost infinite selectivity is possible in using metal(oxide) masks (except $\mathbf{T i}, \mathbf{M o}, \mathbf{W}, \mathbf{N b}$, and $\mathbf{T a}$ which are etching chemically or synergetically); a $10 \mathrm{~nm}$ layer is enough to etch over $400 \mu \mathrm{m}$ in $\mathrm{Si}$, because of the low physical sputtering and -volatility of the metal(oxy)fluorides.

Conductivity: Another important factor to note is the use of insulating masks, because they may be charged to the plasma potential $[3-6]$, thus creating strong local electrical fields at the edge of the mask giving rise to ion bowing and therefore an undercutting directly beneath the mask surface (see fig. 3). An advantage of a mask at plasma potential is its lower mask erosion.

Temperature: When etching $\mathrm{Si}$ trenches it is important that the local temperature of the trench walls is constant, because the surface reactions (adsorption, desorption, et cetera) are highly temperature dependent [11]. For instance, there is some evidence found that eddy currents in the mask, due to the r.f. power source, and/or ion bombardment are increasing the local tem-

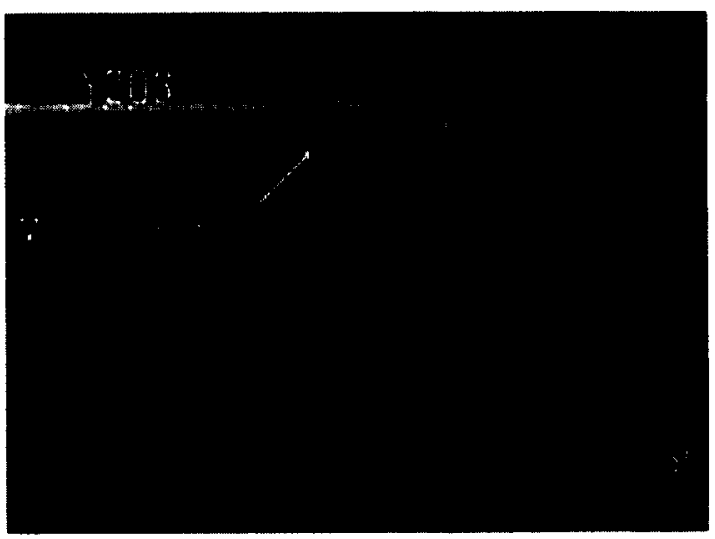

Fig.3: Undercutting of $\mathrm{Y} 203$ masks due to ion bowing. RIE setting: power $=75$ Watt, pressure $=75 \mathrm{mTorr}, \mathrm{SF} 6 / \mathrm{O} / \mathrm{CHF} 3=27 / 11 / 5 \mathrm{sccm}$. 
perature (Joule heat) of the trench walls. This temperature may alter the adsorbility of oxygen at these walls thus changing the thickness of the passivating layer and thus changing the profile. The subject of the temperature dependency of profiles will be treated elsewhere.

Selectivity: It must be stressed that the mask/Si selectivity is strongly depending on the total area of $\mathrm{Si}$ which has to be etched: The $\mathrm{Si}$ etch rate decreases with loading whereas the e.g. $\mathrm{SiO} 2$ etching is barely loading dependent (physical!). In other words; the selectivity is increasing while decreasing the $\mathrm{Si}$ loading.

Photoresist (PR) is the most straightforward mask material. Unfortunately, the PR/Si selectivity is never very high and difficult to control. The main reason is the oxygen (plus fluorine) content in the plasma. Moreover, during etching PR and Si much heat is produced because of the exothermic reactions and therefore the temperature is rising and the selectivity is decreasing (this has its origin in the low glass temperature of $\mathrm{PR}$, making the etching thermo-synergetic). When the temperature is low enough $\left(20^{\circ} \mathrm{C}\right)$ the only etch mechanism is due to impinging ions; a typical ion-enhanced (synergetic) etch mode. The etching of PR is suppressed because of the adsorption of CFx monomers from the CHF3 additive. In short: The etching of PR can be suppressed by 1 . Cooling the substrate by clamping, cryogenically, or by the addition of a cooling gas (e.g. He) in the gas mixture. 2. Lowering the d.c. self bias voltage by changing the reactor geometry (showerhead), an insulating target, or extra dummy $\mathrm{Si}$ in the chamber. 3. Additives such as CFx which don't etch but only compete with the oxygen/fluorine radicals. $\mathrm{SiO} 2$ is another common mask material and is etched synergetically (i.e. chemically $\mathrm{F}^{*}$ under physical control $\mathrm{CFx}+$ ) and therefore the selectivity is limited. As for the PR at low temperature, the etching is ion-enhanced and the selectivity to $\mathrm{Si}$ can be increased by suppressing the d.c. bias. SiNx is even less attractive as a mask than $\mathrm{SiO} 2$, especially when it is not stoichiometric, mainly because it can be etched chemically. Al is IC compatible but, despite of the low energy of the incoming ions (ca. $40 \mathrm{eV}$ ) there is mask erosion (sputtering) visible after greater etch depth ( 20 microns) giving rise to roughening of the $\mathrm{Si}$ adjacent to the $\mathrm{Al}$ protected regions. The erosion may be explained by the existence of eddy currents in the aluminium. Au is even heavier sputtered than $\mathrm{Al}$ (fig.4) may be because it is a very soft noble material. Only at extremely low bias voltages (ca. $15 \mathrm{eV}$ ) resputtering disappears, so the use of a gold mask is limited. Cr seems to be a perfect mask material. It is not resputtered at all at d.c. bias voltages up to $200 \mathrm{eV}$. $Y$ is

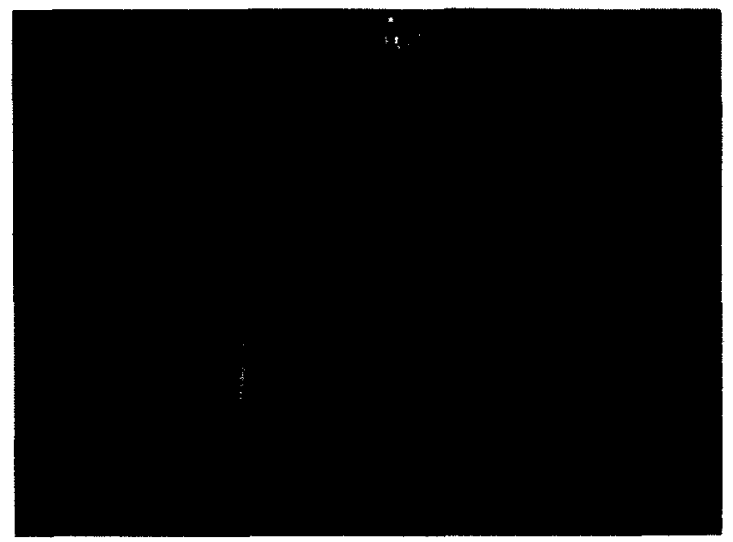

Fig.4: Mask erosion for a gold mask layer at $40 \mathrm{eV}$. RIE setting: power $=75 \mathrm{~W}$, pressure $=75 \mathrm{mTorr}, \mathrm{SF} 6 / \mathrm{O} 2 / \mathrm{CHF} 3=28 / 12 / 0 \mathrm{sccm}$.

easily oxidised in to the strong insulator $\mathrm{Y}_{2} \mathrm{O} 3$ giving rise mask to undercut. Pt, like gold a noble metal, is also easily resputtered although not as strong as gold. $\mathrm{ZnO}$ is a semiconductor which is etched, although not heavily, so its use is limited to low voltages. $\mathbf{P d}$ is an expensive rare earth, but seems to be $\mathrm{OK}$. $\mathrm{Cu}$, although noble, is easily oxidised but gives good results. Ni, like chromium seems to be a very good mask material.

\section{LOADING AND MICROLOADING}

Loading occurs whenever the reactant concentration is depleted due to an excessive substrate load. As a result, the etch rate will decrease inversely proportionally with the $\mathrm{Si}$ area which is exposed to the plasma glow $[9,10]$. At the same time the etched profile will change, because the oxygen/fluorine concentration is altered for a different loading. Another effect which causes the profile to change is the decrease in d.c.bias while increasing the loading, thus decreasing the ion impact. The bias decreases because the etching ions and the reaction products are changing the plasma impedance. An important influence of the loading on the profile is the amount of lateral underetching of the mask (see fig. 1,4). This etching is depending on the thickness of the passivating SiOxFy layer and the fluorine concentration trying to etch the $\mathrm{Si}$ by penetrating this layer. The thickness of the SiOxFy layer is a function of e.g. the oxygen concentration, the ion impact, and the local temperature. The fluorine concentration is a function of e.g. the SF6 flow, power, and (micro)loading; at higher loading there will be less underetching.

When the present process is used in etching $\mathrm{Si}$ structures, the loading should be sufficiently high, e.g. at least $1 \mathrm{~cm}^{2} \mathrm{Si}$, because otherwise the fluorine con- 


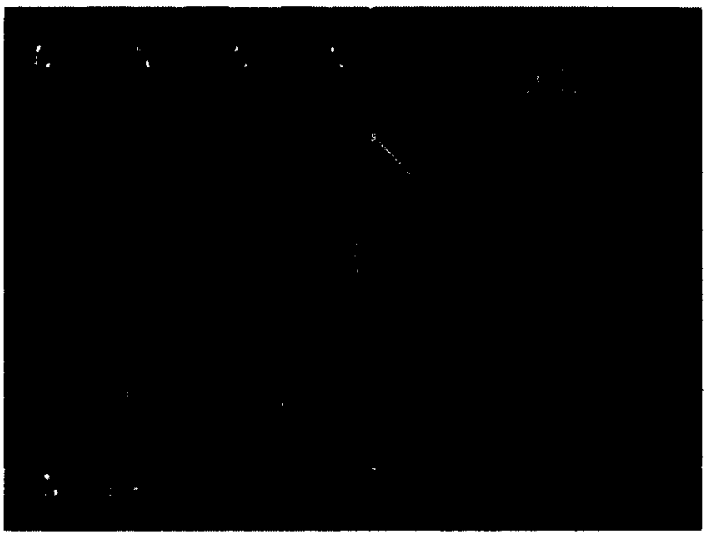

Fig.5: The influence of microloading on the profile. RIE setting: power $=75 \mathrm{~W}$, pressure $=75 \mathrm{mTorr}, \mathrm{SF} 6 / \mathrm{O} 2 / \mathrm{CHF} 3=28 / 5 / 6 \mathrm{sccm}$.

centration at the wafer surface is too high causing underetching. In these cases it is not possible anymore to adjust the parameter setting (pressure, power, or flows) for vertical walls (see the BSM). Nevertheless, it is possible to insert some extra $\mathrm{Si}$ in the reactor in any form to ensure a certain minimal loading.

Microloading is formally equivalent to the loading effect (reactant depletion) and it describes the etching rate dependency on pattern density. It is unlike loading, not a global but a local effect; structures in the locality of big $\mathrm{Si}$ areas are etched at a slower speed than those situated in nonetching areas. This effect is responsible for the strong underetching of the most right finger shown in fig.5. In this picture four polySi combfingers on top of a SiO2 layer are etched. Because of RIE lag, the open area at the right is etched faster than the areas between the fingers where there is still some $\mathrm{Si}$ left. At the moment that the $\mathrm{SiO} 2$ surface is reached for the open area, the local fluorine concentration increases giving rise to an enhanced chemical underetching, indicated by the arrow. This effect can be suppressed by 1 . avoiding RIE lag, 2. defining areas of always etching $\mathrm{Si}$ directly in the neighbourhood of the etching structures, 3. defining walls which can be sacrificed adjacent to the walls (like a windscreen), 4 . changing the plasma chemistry to the point where the passivating SiOxFy layer is thicker, thus decreasing the underetching, or $\underline{\mathbf{5}}$. increasing the total reactor loading with the help of extra dummy Si that much that the overall fluorine concentration is barely changing after the $\mathrm{SiO} 2$ layer is reached.

\section{APPLICATIONS AND CONCLUSIONS}

An important credit of the Black Silicon Method with its diagrams is its ability to control the profile of a $\mathrm{Si}$ trench. In this way all kinds of devices can be created. For instance, while using the BSM we fabricated electrostatically driven comb structures for their use in micro electromechanical systems (MEMS) and tips (radius $<5 \mathrm{~nm}$ !) for AFM applications (eventually integrated with a comb driven XY stage). Black Si can be used directly as an optical diffuser for e.g. laser applications or in sun collectors.

All together, it is shown that the BSM is an excellent tool for finding the dependency of the different plasma etch variables, such as power, pressure, flows, loading, and mask materials. The influence of other important factors on the Si trench etching, such as temperature, target platen, chamber contamination, or wafer holders, will be published elsewhere. Evidence is found that ARDE is controlled by ion bowing; the deflection (and capturing) of impinging ions to the conducting sidewall of a trench. ARDE is found to be suppressed in making the trench walls more insulating-like. It is found that extra $\mathrm{Si}$ in the chamber is an easy way to control the $\mathrm{Si}$ trench profile. The lateral underetching of masks is decreased by increasing the thickness of the SiOxFy passivating layer at the trench wall (>oxygen, <temperature) or decreasing the fluorine concentration (<power, >loading).

\section{REFERENCES}

[1] H.V.Jansen, M.de Boer, R.Legtenberg, M.Elwenspoek, The Black Silicon Method: A Universal method for determining the parameter setting of a fluorine-based RIE in deep Si trench etching with profile control, Proc. Micro Mechanics Europe (MME'94, Pisa, Italy), Sep. 1994, p.60-64, submitted to J.of Micromechanics and Microengineering.

[2] R.Legtenberg, H.Jansen, M.de Boer, M.Elwenspoek, Anisotropic $R I E$ of Si using $S F 6 / O 2 / C H F_{3}$ gas mixtures, submitted to J.Elec.Soc.

[3] R.A.Gottscho, C.W.Jurgensen, and D.J.Vitkavage, Microscopic uniformity in plasma etching, J.Vac.Sci.Tech..B.10(5), Sep.1992.

[4] J.C.Amold and H.H.Sawin, Charging of pattern features during plasma etching, J.Appl.Phys.70 (10), 15 Nov., 1991.

[5] S.G.Ingram, The influence of substrate topography on ion bombardment in plasma etching, J.Appl.Phys. 68 (2), July 1990.

[6] R.H.Bruce and A.R.Reinberg, Profile control with d.c.bias in plasma etching, J.Electrochem.Soc., Vol.129, No 2, Feb. 1982.

[7] W.H.Juan, S.W.Pang, A.Selvakumar, M.W.Putty, K.Najafi, Using ECR source to etch polyimide molds for fabrication of electroplated microstructures, Solid-state S\&A workshop, Hilton Head, South Caroline, June 13-16, 1994.

[8] T.H.Fedynyshyn, G.W.Grynkewich, and T.P.Ma, Mask depen-dent etch rates II, J.Electrochem.Soc., Vol.134, No 10, Oct. 1987.

[9] C.J.Mogab, The loading effect in plasma etching, J.Electrochem.Soc., Vol.124, No 8, Aug. 1977.

[10] T.Enomoto, Loading effect and temperature dependence of etch rate of Si materials in CF4 plasma, Sol. St. Tech., Oct. 1987.

[11] R.N.Carlile, V.C,Liang, O.A.Palusinski, M.M.Smadi, Trench etches in $S i$ with controllable sidewall angles, J. Electrochem. Soc., Vol.135, No.8, Aug. 1988. 